\title{
A Systems Approach to Molecular Electronics
}

\author{
James R. Heath \\ Elizabeth W. Gilloon Professor \\ Caltech Chemistry MC 127-72 \\ 1200 East California Blvd., Pasadena, CA 91125 \\ phone: 626-395-6079 / heath@caltech.edu \\ http://www.its.caltech.edu/ heathgrp/index.htm
}

\section{Abstract}

Molecular electronics is an area of research in which molecules are employed to yield the active and passive device components (switches, diodes, resistors) of an electronic circuit or integrated circuit. Consider the crossbar circuits of nanowires in the electron micrograph at the left [1]. The smallest (100 element) crossbar in this image is patterned at a density approach $10^{12} / \mathrm{cm}^{2}$, and the wire diameter is approximately $8 \mathrm{~nm}$. At a doping level (with species like Boron or Arsenic) of $10^{18} / \mathrm{cm}^{3}$, a similar $8 \mathrm{~nm}$ diameter, micrometer-long segment of silicon wires would have 20-30 dopant atoms, and a junction of two crossed wires would contain approximately 0.1-0.2 dopant atoms. Thus, field-effect transistors fabricated at these wiring densities might exhibit non-statistical, and perhaps nonpredictable behavior. Related arguments, such as the gate oxide thickness, power consumption, (just from leakage currents through the gate oxide), fabrication costs, and others also highlight the difficulty of scaling standard electronics materials to molecular dimensions [2]. The point is that at device areas of a few tens of square nanometers, molecules have a certain fundamental attractiveness because of their size, because they represent the ultimate in terms of atomic control over physical properties, and because of the diversity of properties, such as switching, dynamic organization and recognition that can be achieved through such control.
\end{abstract}

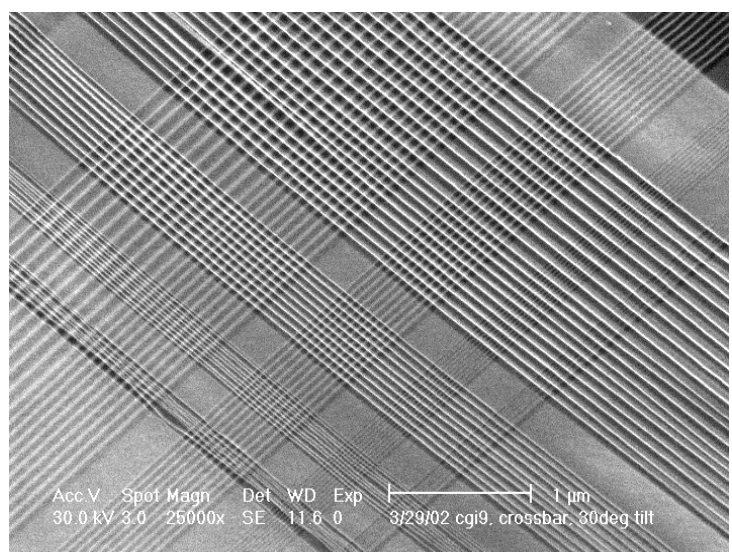

Molecular electronics circuits based on crossbar architectures can be utilized for both logic and memory applications [3], but in order to realize such applications, many things must be simultaneously considered. These include the design of the molecule, the molecule electrode interface, electronically configurable and defect tolerant circuit architectures, methods

Copyright is held by the author/owner(s).

ISLPED'03, August 25-27, 2003, Seoul, Korea.

ACM 1-58113-682-X/03/0008. for bridging the nanometer-scale densities of these circuits to the sub-micrometer densities achievable with lithography, etc. [4] In this talk I will treat such circuits as a system, and discuss how all of these various properties are interrelated. I will also present experimental results of working random-access memory and configurable logic circuits, and FET-based multiplexers capable of bridging length scales.

In these circuits the active device elements are molecular mechanical complexes organized at each of the junctions within the crossbar, as shown at left in the drawing. The molecules are switched via 1 or 2 electron process that results in a mechanical isomerization of the molecule, and thereby a change in the tunneling characteristics of the junction. Detailed electrical measurements on single molecule, three-terminal devices are revealing substantial information concerning how these types of

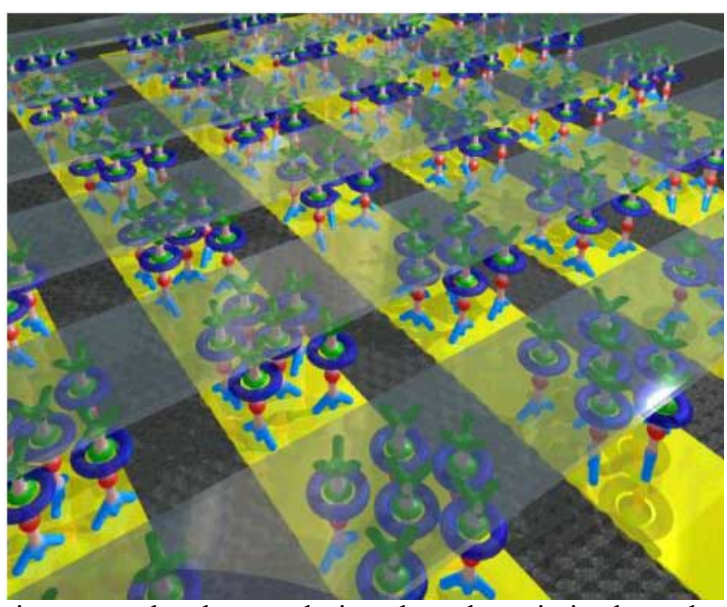

devices can be better designed and optimized, and such measurements will also be presented and discussed.

\section{References}

1. N. Melosh, Akram Boukai, Frederic Diana, Brian Geradot, Antonio Badolato, Pierre Petroff, and James R. Heath, "Ultrahigh density Nanowire Lattices and Circuits," Science, 300, 112 (2003). (WEB published on Science Express 3/14/03).

2. P. Packan, "Pushing the Limits," Science 24, 2079 (1999).

3. Yi Luo, C.P. Collier, Kent Nielsen, Jan Jeppesen, Julie Perkins, Erica DeIonno, Anthony Pease, J. Fraser Stoddart, and James R. Heath, "Molecular Electronics Random Access Memory Circuits," ChemPhysChem 2002(3), 519 (2002).

4. James R. Heath and Mark A. Ratner, "Molecular Electronics," Physics Today, 56, p. 43, May (2003). 